\title{
Improvement of ship stability \& safety in damaged condition through operational measures: challenges and opportunities
}

\author{
Evangelos Boulougouris, University of Strathclyde, UK, evangelos.boulougouris@strath.ac.uk \\ Jakub Cichowicz, Brookes Bell, UK, jakub.cichowicz@brookesbell.com \\ Andrzej Jasionowski, Brookes Bell, Singapore, andrzej.jasionowski@brookesbell.com \\ Dimitris Konovessis, Nanyang Technological University, Singapore, dkonovessis@ntu.edu.sg
}

\begin{abstract}
Guaranteeing a sufficient level of safety from the point of view of stability is typically considered to be a matter of design. The overall level of safety of a ship, however, can only be guaranteed when considering passive design measures in conjunction with active operational measures, in a holistic, balanced and cost-effective manner. Time could therefore be coming for systematically considering operational measures as a recognised and normed integral part of a holistic approach to ship safety from the point of view of stability. In this respect, the scope of this paper is to identify open challenges and to provide, in general, food for thought for stimulating a discussion on the topic of operational measures, with specific attention to the damaged ship condition. The aim is to provide ground for further proceeding towards the goal of implementing a virtuous integrated approach to ship stability safety which gives due credit to effective and robust operational risk control options.
\end{abstract}

Keywords: ship stability; ship dynamics; ship safety; operational measures; damaged condition.

\section{INTRODUCTION}

Required levels of safety with respect to damage ship stability are typically guaranteed by the consideration and evaluation of the effectiveness of proper passive measures at the design stage against applicable regulatory provisions. These measures are in the form of potential design alternatives (hull shape, subdivision, systems redundancy and availability, etc.) and for acceptable loading conditions.

Concerted research and development efforts in the period of the last 30 or so years have mobilised the international maritime community to research on the theoretical understanding of the flooding process and to focus and act on the development of new probabilistic rules for damage stability for all ship types, new ship designs extending and challenging known design limitations, and the Safe Return to Port (SRtP) regulations. Recent related research and development efforts include the activities of the EC-funded research projects SAFEDOR (Breinholf et al., 2009), with developments and refinement of probabilistic methods for the assessment of damage stability and survivability of passenger ships, and GOALDS (Papanikolaou et al., 2013), with significant results in formulating a rational, goal-based regulatory framework properly accounting for damage stability properties of RoPax ships and large cruise ships. Risk-based approaches and cost-effectiveness considerations have been extensively used in this process. A major finding is that the overall level of safety of a ship can only be guaranteed when considering passive design measures in conjunction with active operational measures, in a holistic, balanced and cost-effective manner. 
The concepts of time to flood and time to evacuate and how they interrelate are fundamental notions in determining safety thresholds with respect to ship stability and flooding. Fundamental research on this specific topic has been reported by (Jasionowski et al., 2011) in addressing the compound problem of the absolute time available for passenger evacuation on a damaged RoPax vessel undergoing large scale flooding of vehicle deck spaces. The time for the abandonment of flooded passenger ships due to collision damages, for RoPax and for cruise ships, has been examined by (Spanos and Papanikolaou, 2014). (Vassalos, 2014) details an overall probabilistic framework for flooding risk analysis, time to capsize, time to abandon ship and the evaluation of casualty thresholds/return to port/safety level and their link to systems availability post-casualty. In principle, vulnerability to flooding relates to the cumulative probability for time to capsize in the operational environment of the vessel. This also provides the key input for vulnerability monitoring, which in turn offers all the essential information for damage control and emergency response.

There are therefore many further opportunities for research and development associated with the idea of giving a more systematic and quantifiable importance to operational measures. At the same time, however, there are also numerous challenges. Some ideas regarding opportunities and challenges have been collected in the following, where the material is split in three sections, namely: operational guidance and procedures; systems availability post-damage; active measures for damage containment. It is noted that operational guidance and system availability post-damage relate to maintaining the design safety envelop through the monitoring and decision-support, facilitating operational intervention. In this paper, we provide elaborations on open challenges and food for thought for elaborating on the topic of operational measures, with specific attention to the damaged ship condition.

\section{OPERATIONAL GUIDANCE AND PROCEDURES}

Technological advances in computing hardware over the last decades have facilitated solution of many problems in ever decreasing amount of time. However, the progress in technical calculus, involving modelling based on the fundamental physical laws, has been just as significant, and despite the availability of ever grater processing power, many cases of numerical approximations to reality remain impractical to compute. It is for this reason that advanced prognosis have only had limited success in proliferating the field of instantaneous decision support.

Although highly advanced computerised safety management systems (SMS), have found accelerated support, their advisory functionality are mostly limited to detection only, with more sophisticated prognosis and advisory capabilities remaining at prototyping and development stages.

Such prototype simulation approaches available for use in prognosis comprise a range of phenomena such as (a) ship response to flooding progression, modelled through various but direct solution to conservation of momentum laws, or through quasi-static iterative approximations, (b) structural stress evolution under flooding, (c) the mustering process, (d) fire and smoke spread, and possibly many other.

Some of the reasons inhibiting their more wide use for decision support arise due to a series of practical problems in addition to sheer computational effort, such as the following:

- Each of these processes may vary at any instant of time due to changing conditions.

- The input is subject to considerable uncertainty.

- For any set of input information the outcome is random due to computational and modelling uncertainties as well as due to random nature of environmental or process conditions themselves. 
- Each may be seriously influenced by decision choices.

The nature as well as inseparable combination of these engineering challenges imply that the projection functionality would need to be iterated for a range of uncertain conditions of either of the scenarios occurring as well as for a range of decision options, so that the best choice can be identified with controllable degree of confidence. This, in turn, implies that the computational task of scenario projection in real time in support of decision making will likely remain a serious challenge, as most of these analyses require substantial amount of processing time, at present accounted in hours.

Vulnerability Log, or VLog for short, has been proposed to be the functionality to inform the crew at all times on the instantaneous vulnerability to flooding of the vessel, considering its actual loading conditions, the environmental conditions and the actual watertight integrity architecture (Jasionowski, 2011). The vulnerability is proposed to be measured in terms of the probability that a vessel might capsize within given time when subject to any feasible flooding scenario. Figure 1 illustrates a sample screenshot of vulnerability information, whilst Figure 2 demonstrates the distribution of vulnerability logged on a demonstration ship.

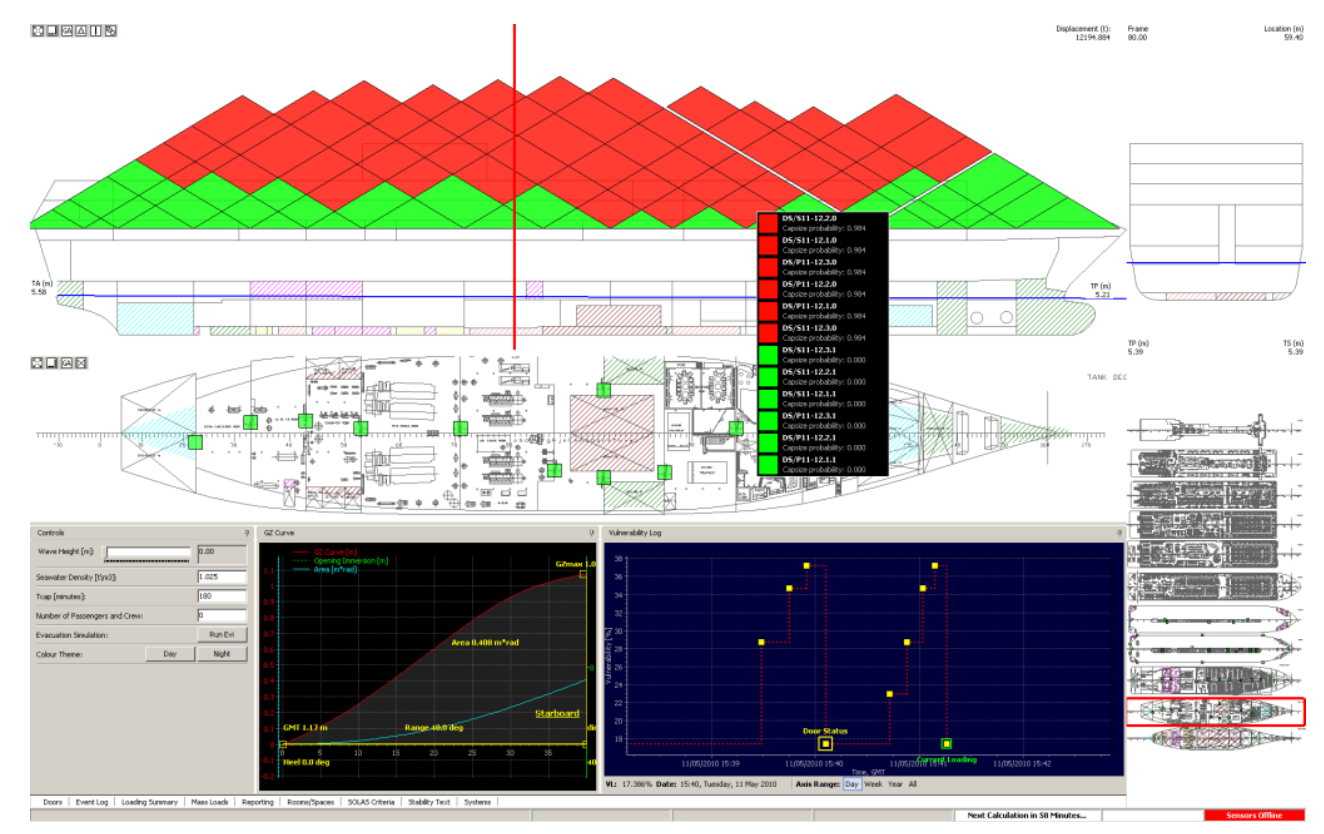

Figure 1: Sample screenshot for ship overall vulnerability. 


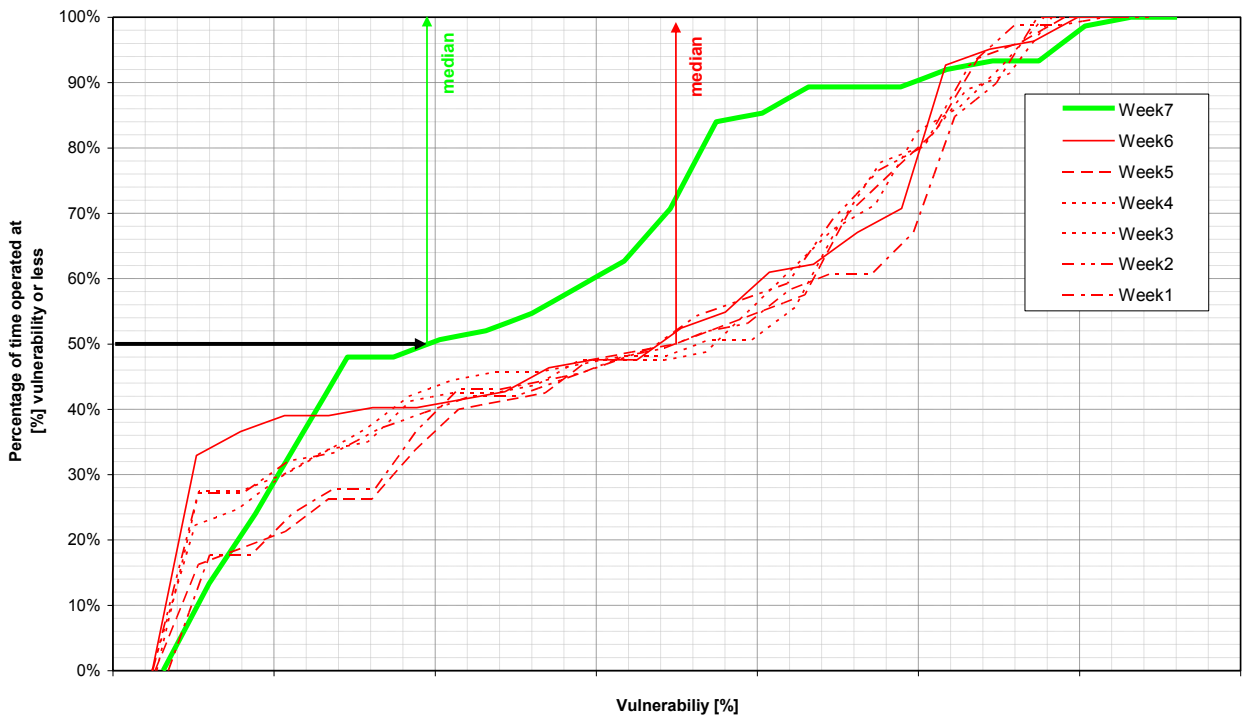

Figure 2 Distribution of vulnerability logged on a demonstration ship. The actual vulnerability values are undisclosed. The impact of the awareness of the crew on the day-to-day management of watertight integrity, and hence crew and ship preparedness, can be seen in Week 7, when explanation and training on use of VLog had been given.

Since until a casualty occurs it is impossible to anticipate any specifics of a flooding case a ship might suffer and therefore let the crew prepare for it, it seems plausible that instead the crew is made aware of the range of such flooding specifics together with projected impact these can have on the ship state. The crew would be able to infer the criticality of the situation evolving immediately, based on their own awareness, and hence decide instinctively of the best possible actions to follow. Ship vulnerability to flooding will naturally vary significantly from a flooding case to a flooding case, and subject to what condition the vessel operates at, at which environment and what is the watertight integrity status. All these must, therefore, be considered.

The framework for vulnerability assessment given in (Jasionowski and Vassalos, 2006) can serve as a very informative model for use in the context of decision making. It reflects fundamentals of physical processes governing ship stability in waves and explicitly acknowledges uncertainty of such predictions by exploiting probability theory.

Therefore, further research efforts should be expanded to establish and verify practicalities of the principles of the proposed functionality, as well as to assess impact of all engineering approximations that are used in application of the proposed model. Many such aspects should be considered, with key focus on uncertainty in the widest sense, pertaining to its both aleatory as well as epistemic types. Example impact of treatment of actual tank loads in assessing stability, effects of damage character, relative importance of transient flooding stages, accuracy of physical experimentation used as basis data, or simple elements such as effect of computational speed on functionality of the whole proposition, or ergonomics of the conveying techniques used. The prime objective is to find solutions acceptable for wider industrial application, taking into account that in a real emergency there will be a very limited number of possible emergency scenarios, or even a single emergency scenario, hence making it easier to evaluate and address. Thus, in real life emergency situations effective vulnerability monitoring can be achieved by the assessment of KPIs relating to safety and performance, putting emphasis on assessing "what went wrong" and "how it can be fixed" and not 
on "why it went wrong". A recent brainstorming workshop for applicable design and operational measures for flooding emergency incidents onboard RoPax ships, initiated by INTERFERRY Europe and reported by (Vassalos et al., 2014), has produced a comprehensive matrix of accidental flooding events and related means for risk reduction in terms of reduction of the likelihood and/or the severity of the consequences of a flooding event in association with risk contributing factors.

\section{SYSTEMS AVAILABILITY POST-ACCIDENT}

Formally, the safe return to port (SRtP) regulations adopted at $82^{\text {nd }}$ session of MSC and subsequent amendments to SOLAS are not linked to damage stability and although it makes little sense to speculate about the reasons behind the separation, the formal disengagement by IMO seem to be utterly intentional. Nevertheless, the separation does not undermine the strong and authentic interrelation between the damage stability framework and SRtP, at least in part of the latter referring to flooding casualties. In short, all SRtP-compliant vessels need to demonstrate that their safetycritical systems remain operational outside the casualty area following single-compartment flooding. That is, the SRtP capability is to be demonstrated for specific subset of all possible flooding scenarios.

It is in fact the way the subset of flooding scenarios is being defined that prevents harmonisation of SRtP with damage stability framework. The SRtP subset is deterministic while the damage stability calculations draw from probabilistic domain (Cichowicz et al., 2009), (Dodman, 2010). For this reason while the damage stability framework is differentiating the flooding damages due to their likelihood (based on the extent), the SRtP is not. One of the consequences of this is that the SRtP regulations are inevitably confined to small-extent damages. At a glance, such scenarios seem to form an intuitive domain for considering the post-casualty capability for sailing under own-power (one can reason that realistic conditions for SRtP would at least require list/trim ensuring sufficient residual stability, calm seas and reduced speed). However, as the availability assessment take no notice of the ship's survivability in flooding scenarios, hence certain systems, such as propulsion, may be required to remain operational even in the scenarios leading to rapid ship loss due to floatability or stability failures (it is not uncommon for SOLAS compliant ships to be vulnerable to single-watertightcompartment flooding cases). On the other hand the SRtP framework completely disregards significant number of probable (and survivable) multiple-compartment flooding scenarios in which even availability of systems critical for evacuation and abandonment are not assessed. This results in serious inconsistence between the frameworks that seriously undermines significance of the SRtP. Furthermore, neglecting the information about likelihood of casualty within the specific space prevents (or at least complicates) protecting the systems by design, i.e. locating critical components and routing critical connectors through the locations of low probability of being affected by a casualty.

As mentioned in the foregoing exact reasons for which these two frameworks have been severed is unclear but one of the reasons for this may be related to complexity of the assessment process. In essence, the SRtP require the assessment to be performed on system models embedded within the vessel arrangement including both WT subdivision and A-class boundaries, with the latter setting a minimum spatial resolution of the models. 


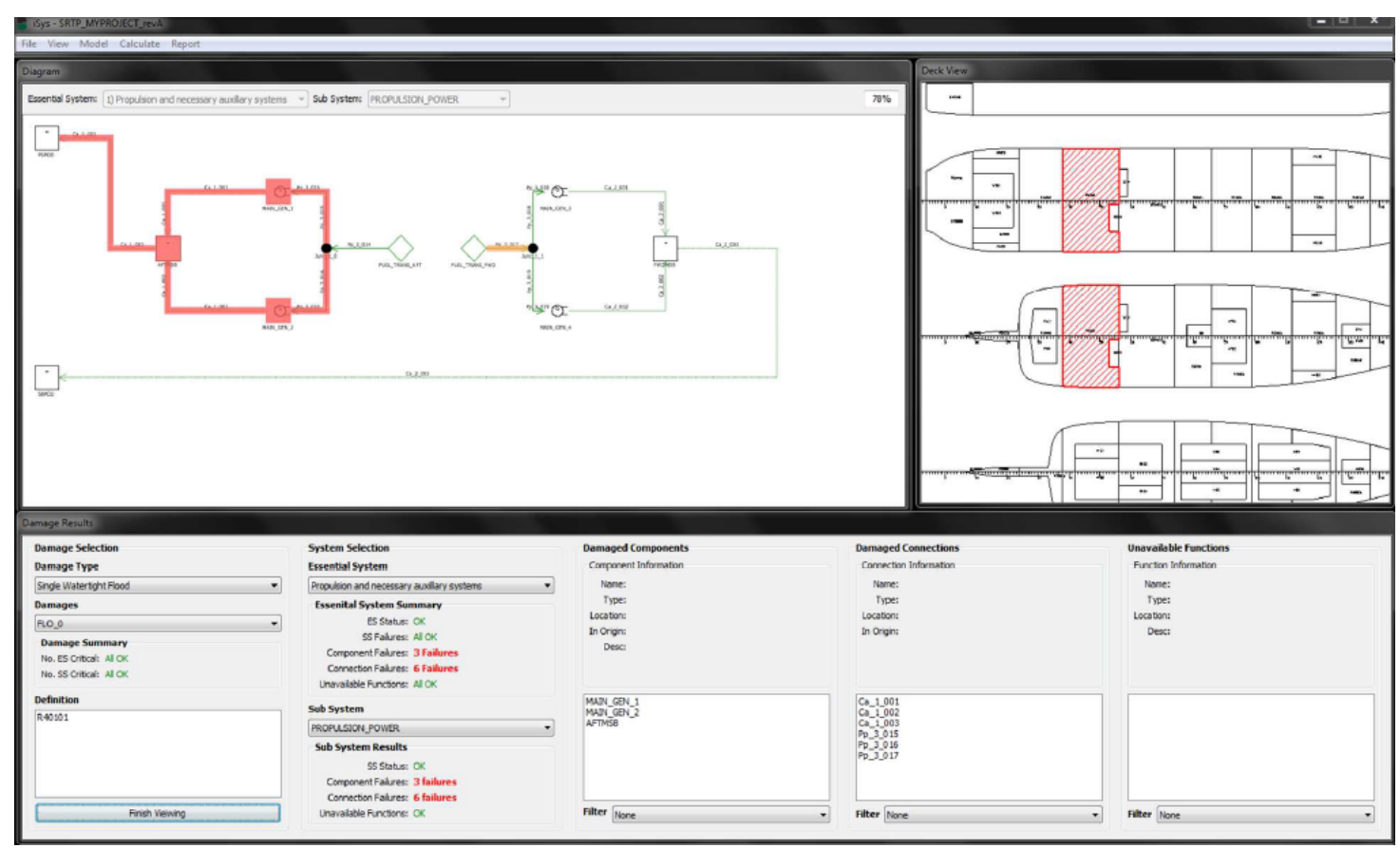

Figure 3: The iSys package by Brookes Bell/Safety at Sea allows for rapid modelling of complex systems embedded in ship's arrangement. The tool allows for assessing post-casualty availability of the systems, creating FMEA-style tables and is capable of generating recommendations for restoring of systems' functionality.

Furthermore, experience shows that identification of vulnerabilities flaws in typical plant design requires modelling not only the main systems but also many auxiliary and peripheral systems (i.e. control, monitoring, remote access, etc.) in order to capture the fine details of the functionality. The design principles of ship systems are well-established and backed by long experience, hence in principle the onboard system are equally sound and have sufficient level of built-in redundancy. Yet, the complex system often suffer from well-hidden deficiencies resulting in serious vulnerabilities to even minor flooding or fire accidents (as observed during some quite-recent incidents on cruise ships).

The problem of such concealed vulnerabilities is particularly important in case of passenger ships (of ever-growing capacity and sailing to the most remote corners of the oceans) and the offshore production plants (where again the isolation and accessibility of remote assistance becomes a serious issue). Yet, even in application to very complex plants the interaction between stability and SRtP is unidirectional with components of the flooding risk models affecting the actual SRtP capability (even if that information is largely lost in the IMO implementation) but not vice versa. However, recent research in the field of damage stability (see for example project GOALDS) show clearly that although there is still room for improving accuracy of individual constituents (such as por s-factors) of the flooding risk models there is also a ceiling in cost-effectiveness of passive riskcontrol options. In other words, the structural means of providing buoyancy and stability (i.e. subdivision and distribution of compartments within the watertight envelope) become expensive (in terms of either build or running costs) and ineffective in designs aiming at high attained index of subdivision (A-index).

This renders the concept of the required index $\mathrm{R}$ equalling 1 commercially unattainable without use of active stability-enhancing devices. Inevitably, risk reduction coming from operation of active measures would be conditional on their post-casualty availability which should be assessed analogically to SRtP performance. 


\section{ACTIVE MEASURES FOR DAMAGE CONTAINMENT}

Since the advent of the damage stability requirements, the reduction of the severity of the consequences of a flooding event was synonym to the enhancement of the internal watertight subdivision of the ship. As also mentioned in the foregoing, there has been considerable research regarding the improvements that the designer may achieve by proper subdivision. These improvements come with a cost such as the complexity of the internal arrangement and the reduction of the ergonomics, the additional weight penalties and the reduction the payload of the vessels. Therefore, many have argued that we are approaching a plateau i.e. a stage that no further vulnerability enhancements may be expected by subdividing further the internal volume of the ship.

One should notice that the subdivision is an inherent characteristic of the design and in that respect a "passive" way of improving the survivability of the ship after damage. This was mainly stipulated by the fact that the international community was always very sceptical regarding the feasibility and the reliability of active measures as in many accidents active systems related to the safety of the ship failed to operate as planned (e.g. emergency generators, fire-fighting systems etc.).

On the other hand, it is obvious that technology has advanced significantly since the loss of the SS Titanic and SOLAS 1914 and our understanding of the physics of ship's flooding, even in waves has come a long way even since the loss of MS Estonia twenty one years ago. In this respect, it seems from technological point of view, increasingly more appealing and from the industry point of view more demanding to start considering new, more active ways of reducing the severity of consequences of a flooding event. Such measures could be of operational nature and/or active measures and they should be considered in addition to design (passive) measures. The justification for using such measures should be based on a cost-benefit analysis according to the level of risk reduction achieved. IMO Circular 1455 on Alternatives and Equivalents provides a the basis for such a consideration but there are practical and philosophical problems to be overcome regarding the "summing up" of the risk reduction from design and operational means.

An integrated method for the management of the vulnerability has been proposed by (Vassalos and Jasionowski, 2013) shown in Figure 4. It brings together the traditional risk control measures focusing on design solutions (category 1), with the operational/active measures (category 2) and the emergency response measure/systems (category 3). Whilst SOLAS Ch. II-2 recognizes now the significant of active measures such as damage control, such measures have not been validated to the same level of rigour as category 1 measures. Furthermore, for many of the category 3 measures, such as Decision Support Systems for Crisis Management, Evacuation, LSA, Escape and Rescue, the costeffectiveness of their risk reduction potential has never been measured nor verified. In many accidents it can be argued that the fatalities would be significantly less if this second barrier of defence by category 2 measures was more developed. 


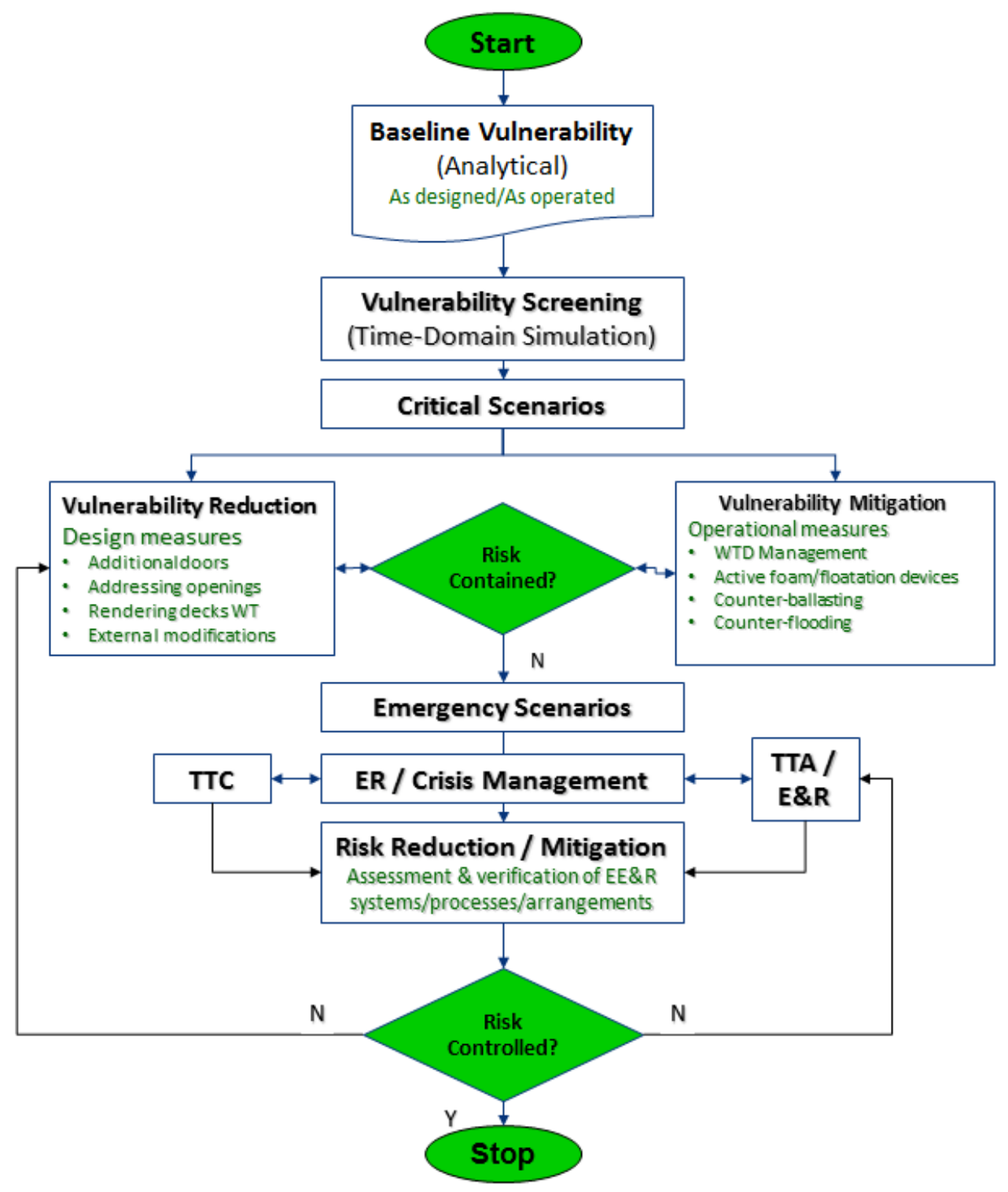

Figure 4: Vulnerability Management

Such a system is the Damage Stability Recovery System (DSRS). It can be fitted to new or retrofitted existing RoPax in order to reduce the likelihood of capsize/sinking and further water ingress following a major incident / accident (Vassalos, 2015). The proposed system uses foaming agents distributed along the ship by a dedicated piping system with its own pumps. The safety-critical compartments are identified and the foam is delivered to them through dedicated nozzles. The system is able to withstand the ingress water pressure and provides a void filling mechanism to reduce flooding and thus enhance the buoyancy and stability of the vessel. The use of the system is under the full control of the crew, with a decision support system (DSS) available to help the ship officers decide where and when the system will act as well as inform them of the ensuing effect. The system complies with identified requirements for the timely delivery of the foam in the damaged compartments to prevent progressive flooding and stability loss.

The foam itself meets all the environmental and health criteria, it is not toxic to humans and its release does not pose any danger to the people onboard or the environment. The system is illustrated in Figure 5. 
Key characteristics of the system include:

- Modular design

- Piping system running along the centre of the vessel

- Nozzles located in each of the primary spaces

- Non-intrusive:

- Optimum location in vessel - "void", "out of the way" spaces.
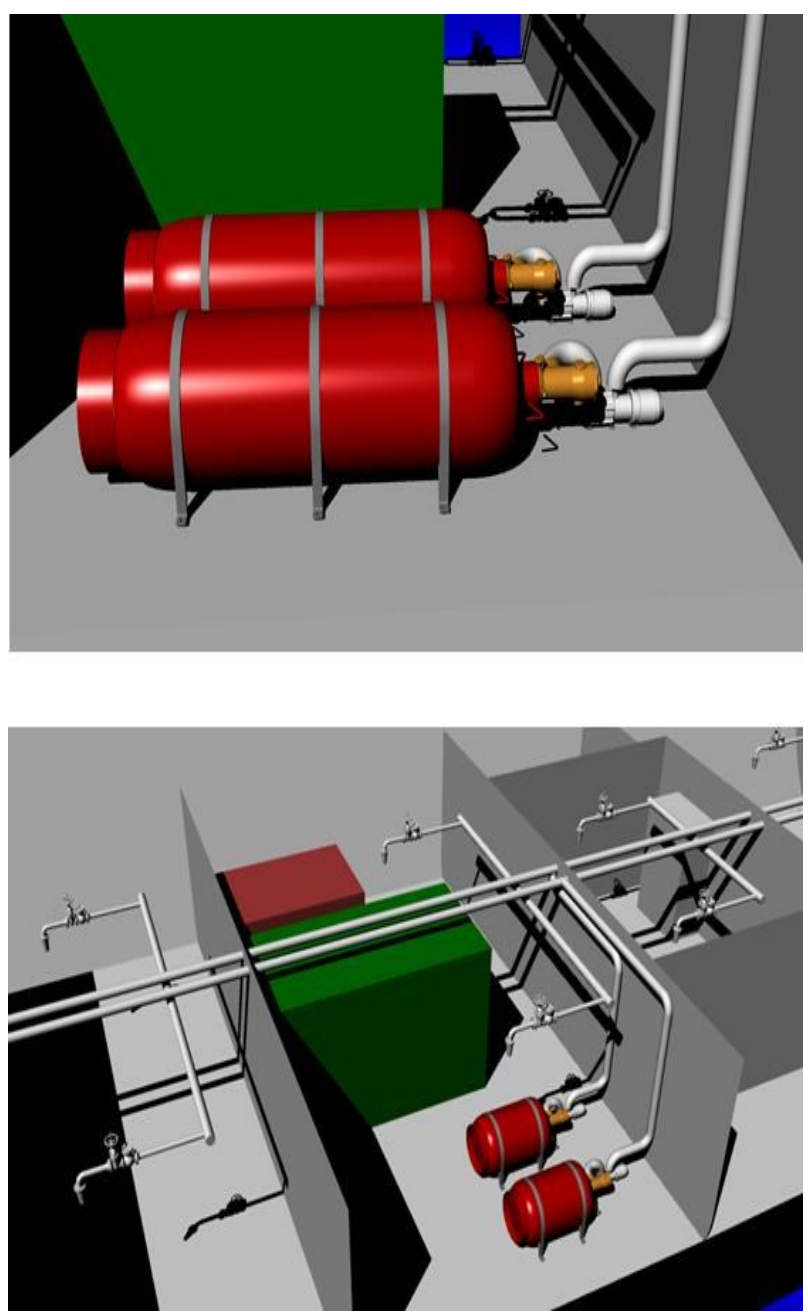

Figure 5: Damage Stability Recovery System (DSRS)

\section{FINAL REMARKS}

In this paper, we provided some elaborations on the current state-of-affairs with regards to operational measures relating to damage stability and safety. The aim was to provide ground for further proceeding towards the goal of implementing a virtuous integrated approach to ship stability safety which gives due credit to effective and robust operational risk control options.

\section{ACKNOWLEGEMENTS}

This collaborative effort has been undertaken by members of the "Stability R\&D Committee" (www.shipstab.org/stability-r-d-committee-srdc/). The authors would like to express their sincere 
thanks to the organisers of the $12^{\text {th }}$ International Conference on Stability of Ships and Ocean Vehicles (STAB 2015, Glasgow, UK) for the opportunity given to present this work.

\section{REFERENCES}

Breinholt, C., Ehrke, K.C., Pavaut, C., Sames, P.C., Skjong, R., Strang, T., Vassalos, D., 2009. "SAFEDOR - The Implementation of Risk-Based Design and Approval", Proceedings of the $10^{\text {th }}$ International Marine Design Conference (IMDC 2009), 26-29 May 2009, Trondheim, Norway, pp. 697-704.

Dodman, J., 2010. "Going Forward with Safe Return to Port", Proceedings of the $11^{\text {th }}$ International Ship Stability Workshop, Wageningen, The Netherlands, pp. 32-37.

Cichowicz, J., Vassalos, D., Logan, J., 2009. "Probabilistic Assessment of Post-Casualty Availability of Ship Systems", Proceedings of the $10^{\text {th }}$ International Conference on Stability of Ships and Ocean Vehicles (STAB 2009), St. Petersburg, Russia, pp. 453-462.

Jasionowski, A., Vassalos, D., 2006. “Conceptualising Risk", Proceedings of the $9^{\text {th }}$ International Conference on Stability of Ships and Ocean Vehicles (STAB 2006), Rio de Janeiro, Brazil, pp. 97109.

Jasionowski, A., 2011. "Decision Support for Ship Flooding Crisis Management", Ocean Engineering, Volume 38, pp. 1568-1581.

Jasionowski, A., Vassalos, D., Guarin, L., 2011. "Time-based Survival Criteria for Passenger Ro-Ro Vessels", in Contemporary Ideas on Ship Stability and Capsizing in Waves, M.A.S. Neves et al. (eds.), pp. 663-687.

Papanikolaou, A., Hamann, R., Lee, B.S., Mains, C., Olufsen, O., Vassalos, D., Zaraphonitis, G., 2013. "GOALDS - Goal Based Damage Ship Stability and Safety Standards", Accident Analysis and Prevention, Volume 60, pp. 353-365.

Spanos, D., Papanikolaou, A., 2014. "On the time for the abandonment of flooded passenger ships due to collision damages", Journal of Marine Science and Technology, Volume 19, Issue 3, pp. 327337.

Vassalos, D., Jasionowski, A., 2013. "Emergency Response in Flooding Casualties", 13th International Ship Stability Workshop, Brest, France, 21-26 September 2013, pp. 259-263.

Vassalos, D., 2014. "Damage Stability and Survivability - 'Nailing' Passenger Ship Safety Problems", Ships and Offshore Structures, Volume 9, Issue 3, pp. 237-256.

Vassalos, D., Boulougouris, E., Guarin, L., Jasionowski, A., Garner, J., 2014. "Regulatory, Design, Operational and Emergency Response Measures for Improving the Damage Survivability of Existing RoPax", Proceedings of the $14^{\text {th }}$ International Ship Stability Workshop (ISSW 2014), 29 September to 1 October 2014, Kuala Lumpur, pp. 292-300.

Vassalos, D., 2015. "Life-Cycle Vulnerability Management of RoRo Passenger Ships", Damaged Ship III, RINA International Conference, London, UK, March 2015. 
